\title{
The Habitable Zone and Extreme Planetary Orbits
}

\author{
Stephen R. Kane and Dawn M. Gelino
}

\begin{abstract}
The habitable zone for a given star describes the range of circumstellar distances from the star within which a planet could have liquid water on its surface, which depends upon the stellar properties. Here we describe the development of the habitable zone concept, its application to our own solar system, and its subsequent application to exoplanetary systems. We further apply this to planets in extreme eccentric orbits and show how they may still retain life-bearing properties depending upon the percentage of the total orbit which is spent within the habitable zone. Key Words: Extrasolar planets-Habitable zone-Astrobiology. Astrobiology 12, 940-945.
\end{abstract}

\section{Introduction}

T HE DETECTION OF EXOPLANETS has undergone extraordinary growth over the past couple of decades since substellar companions were detected around HD 114762 (Latham et al., 1989), pulsars (Wolszczan and Frail, 1992), and 51 Peg (Mayor and Queloz, 1995). From there the field rapidly expanded from the exclusivity of exoplanet detection to include exoplanet characterization. This has been largely facilitated by the discovery of transiting planets, which allows unique opportunities to study the transmission and absorption properties of their atmospheres during primary transit (Knutson et al., 2007; Agol et al., 2010) and secondary eclipse (Charbonneau et al., 2005; Richardson et al., 2007). In addition, studies of phase curves allow insight into the thermal and albedo atmospheric properties (Kane and Gelino 2010, 2011). These studies are being applied to smaller planets as our detection sensitivity pushes down into the super-Earth regime (Bean et al., 2011; Croll et al., 2011).

The expansion of exoplanetary science is leading the field toward the question of the frequency of Earth-like planets and, in particular, of habitable planets. The merging of stellar and planetary properties results in a quantitative description of the habitable zone (HZ), usually defined as that region around a star where water can exist in a liquid state. Although habitability had certainly been considered before, the concept of a HZ first started to appear in the science literature during the 1950s in articles such those by Su-Shu Huang $(1959,1960)$. However, these were written at a time when atmospheric studies of other planets were in their infancy and the presence of planets outside our solar system had not yet been confirmed. The work of Kasting et al. (1993) was instrumental in new advancements in $\mathrm{HZ}$ research and in particular quantifying the boundaries in terms of climate models as well as the properties of the host star.

Here we outline the development of the HZ and the factors which influence the boundary conditions. We apply this to our solar system and discuss the extension to exoplanetary systems. We also discuss highly eccentric orbits and how the time spent within the $\mathrm{HZ}$ affects the conditions for habitability and extremophiles that could survive under these conditions.

\section{Habitable Zone Boundaries}

The boundaries of the HZ for a particular system are calculated based upon both the stellar properties and assumptions regarding the response of a planetary atmosphere to stellar flux. Calculations for $\mathrm{HZ}$ boundaries have undergone considerable change since the published articles of Huang $(1959,1960)$. Estimates for our solar system by Dole and Asimov (1964) were in the relatively broad range of 0.725-1.24 AU, compared with the conservative estimates by Hart (1979) of 0.95-1.01 AU. Detailed models for runaway greenhouse implications for Venus were considered by Pollack (1971) and Kasting (1988). For more recent studies regarding the HZ, we refer the reader to the detailed models of Kasting et al. (1993), Kasting and Catling (2003), Underwood et al. (2003), Selsis et al. (2007), and Jones and Sleep (2010). Here we describe the primary factors which influence the boundaries and present examples both in and out of our solar system.

The premise that remotely detectable habitability requires water to exist on a planetary surface in a liquid state allows a 


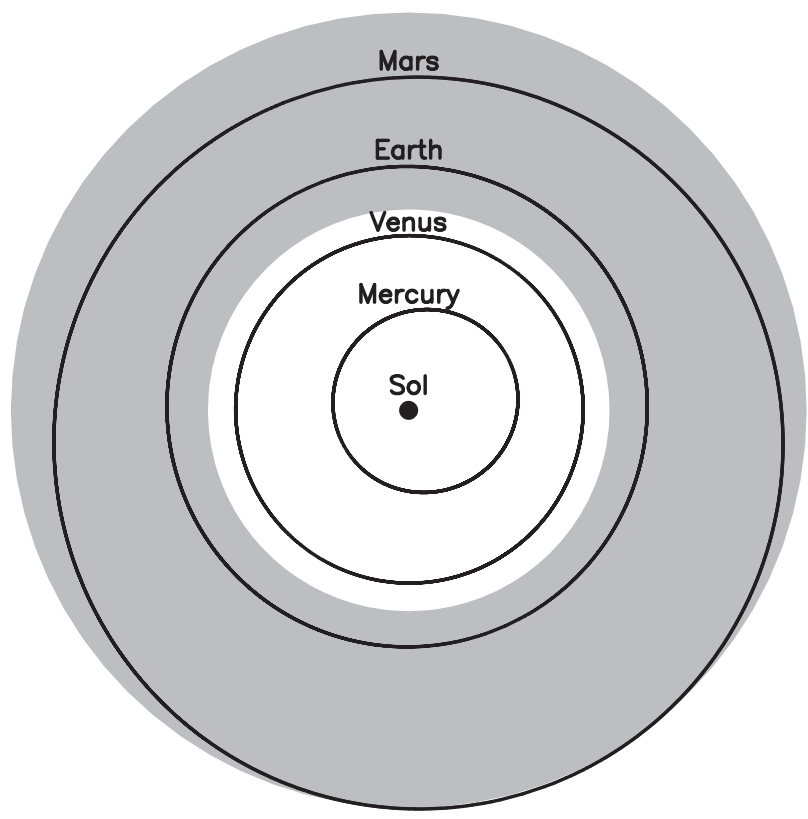

FIG. 1. The inner Solar System showing the orbits of the terrestrial planets. The HZ is indicted by a gray disk.

quantitative representation of the $\mathrm{HZ}$ to be estimated. This is established by combining the stellar properties with those of a hypothetical planet at various distances from the star. The flux received by the planet is a function of this distance and the luminosity of the star. Kasting et al. (1993) used onedimensional (altitude) climate models to calculate the atmospheric response of the atmosphere to the flux by considering conditions whereby the equilibrium would sway to a runaway greenhouse effect or to a runaway snowball effect. The runaway greenhouse cycle is summarized as follows: increased $\mathrm{CO}_{2}$ and/or increased stellar flux; temperature increases; temperature rise leads to an increase in water vapor; increased greenhouse gas concentration leads to high temperatures. Note that the increase in water vapor leads to rapid loss of hydrogen and increased absorption at the wavelengths through which much of Earth's infrared radiation escapes, thus propagating the runaway warming of the surface. The runaway snowball model is summarized as follows: reduced greenhouse gases and/or reduced stellar flux; temperature decreases; resulting ice increases surface reflectivity; reduced heating leads to further ice formation. Note that this also results in significant amounts of atmospheric $\mathrm{CO}_{2}$ condensing as dry ice and thus further reducing the greenhouse effect.

The overall habitability is further complicated by the various other factors inherent to the system. The flux from the host star depends on spectral type and luminosity class but also depends on the age of the star. As the age of the star increases, so does the luminosity, and thus the HZ moves outward from the center of the system. One then can consider a continuously habitable zone in which a particular planetary orbit may remain while the star is on the main sequence, since the movement of the $\mathrm{HZ}$ continues even after the star leaves the main sequence (Lopez et al., 2005). As described above, the planetary atmospheric and albedo properties play an important role in controlling the heat re-
Table 1. Calculated Blackbody Temperatures for the Terrestrial Planets Assuming Complete

Heat Redistribution (Temp 1) and No Heat Redistribution Resulting in a Hot Dayside (Temp 2)

\begin{tabular}{lccc}
\hline Planet & Temp 1(K) & Temp 2 (K) & Actual Temp (K) \\
\hline Mercury & 440 & 523 & 440 \\
Venus & 184 & 219 & 737 \\
Earth & 254 & 302 & 288 \\
Mars & 210 & 250 & 210 \\
\hline
\end{tabular}

For comparison, we show the actual measured mean surface temperatures.

tained at the surface. The redistribution of that heat is in turn related to the planetary rotation rate, particularly important for planets in the HZ which may be tidally locked around late-type stars (Williams and Kasting, 1997). In addition, the planetary mass will influence such aspects as the amount of retained atmosphere and the level and continuity of volcanic activity. Too small a mass will likely lead to a lack of these attributes, and too high a mass will lead to a possibly inhospitable thick hydrogen envelope that will further obscure the visibility of biosignatures. Thus, optimal planetary masses exist which result in a more suitable balancing of these effects.

In the following sections, we apply the equations of Underwood et al. (2003) and Jones and Sleep (2010), which relate the HZ boundaries to stellar luminosity and effective temperature for reasonable models of planetary atmospheric responses.

\subsection{Application to the Solar System}

By way of demonstration, we first apply the HZ models to our own solar system. For a solar effective temperature of $5778 \mathrm{~K}$, we find the inner and outer boundaries of the HZ to be 0.836 and $1.656 \mathrm{AU}$, respectively. Figure 1 shows the $\mathrm{HZ}$ depicted as a gray disk with the orbits of the terrestrial inner planets overlaid.

An estimate of the equilibrium temperatures for the inner planets can be calculated by using their known orbital parameters and Bond albedos ${ }^{1}$ and treating them as blackbodies, as described by Kane and Gelino (2011). This calculation is further explored by considering two cases: one in which the heat is evenly redistributed throughout the surface and the other where the heat redistribution is restricted to the dayside of the planet. The results of this are shown in Table 1, where Temp 1 is the former and Temp 2 is the latter. This shows that the model of complete heat redistribution works reasonably well for Solar System terrestrial planets. The major exception to this is Venus, whose temperature has become dominated by the climate changes that have resulted in a runaway greenhouse effect, as described earlier. For Mercury, the actual surface temperature varies enormously and is highly position-dependent on the planet due to tidal locking and the lack of any substantial atmosphere. For Earth, the calculated temperature of $254 \mathrm{~K}$ compared with the actual temperature of $288 \mathrm{~K}$ reveals an

\footnotetext{
${ }^{1}$ http://nssdc.gsfc.nasa.gov/planetary/factsheet.
} 
inability of this simple model to correctly account for atmospheric (greenhouse) warming of the surface. Thus one should be cautious when using such a model to estimate temperatures of terrestrial-sized planets in exosystems since there can potentially be a large range of temperatures spanned by uncertainties in albedo and greenhouse effect.

\subsection{Application to exoplanets}

These techniques may be applied to known exoplanetary systems for which there are a variety of host stars and associated planets. There have been numerous recent discoveries of super-Earth-mass planets whose orbits have been found to lie within their stars' HZ. Examples include Gliese 581 d (von Paris et al., 2010; Wordsworth et al., 2011), which is in a multiplanet system around an M dwarf, and the more recent case of a potentially habitable planet around HD 85512 (Kaltenegger et al., 2011). The investigation of the HZ for Kepler candidates is of particular interest since many of these planets fall in the terrestrial regime (Kaltenegger and Sasselov, 2011).

Many of the known exoplanets are of jovian mass; thus we do not necessarily consider them as habitable on their own. However, it is worth considering that these planets likely harbor their own systems of terrestrial moons considering the frequency with which we see such occurrences in our own system. Indeed one may consider how the ecology of the jovian moons may have evolved, were Jupiter in Earth's current orbit. The habitability of exomoons has been considered in the literature (Kaltenegger, 2010; Porter and Grundy, 2011), and attempts are being undertaken to detect their presence from Kepler mission data (Kipping et al., 2009, 2012). There are also alternative energy sources for exomoons, such as tides, which may help or hinder habitability and even allow an extension of habitable conditions beyond the traditional HZ (Williams et al., 1997; Scharf, 2006; Barnes et al., 2009).

\subsection{The Habitable Zone Gallery}

In order to provide an easily accessible service to the astronomical community for the investigation of $\mathrm{HZ}$ information for known exoplanetary systems, we developed the Habitable Zone Gallery (www.hzgallery.org). This service provides HZ details for each of the exoplanetary systems with sufficiently known planetary and stellar parameters to carry out the necessary calculations. This catalogue is regularly updated as new discoveries are made and new data becomes available. The service includes a sortable table with information on the percentage of the orbital phase each exoplanet spends within its HZ, planetary effective temperatures, and other basic planetary properties. In addition to the table, the service provides a gallery of known systems, which plots the orbits and the location of the HZ with respect to those orbits, such as that seen in Fig. 1, and movies that animate the movement and temperature of a planet as it orbits the star. A summary plot shows the relation between period, orbital eccentricity, and the percentage time spent in the HZ. The underlying calculations and infrastructure for the Habitable Zone Gallery are described in detail by Kane and Gelino (2012). In the following section we consider extreme cases of eccentric orbits whose path takes the planet through the HZ.

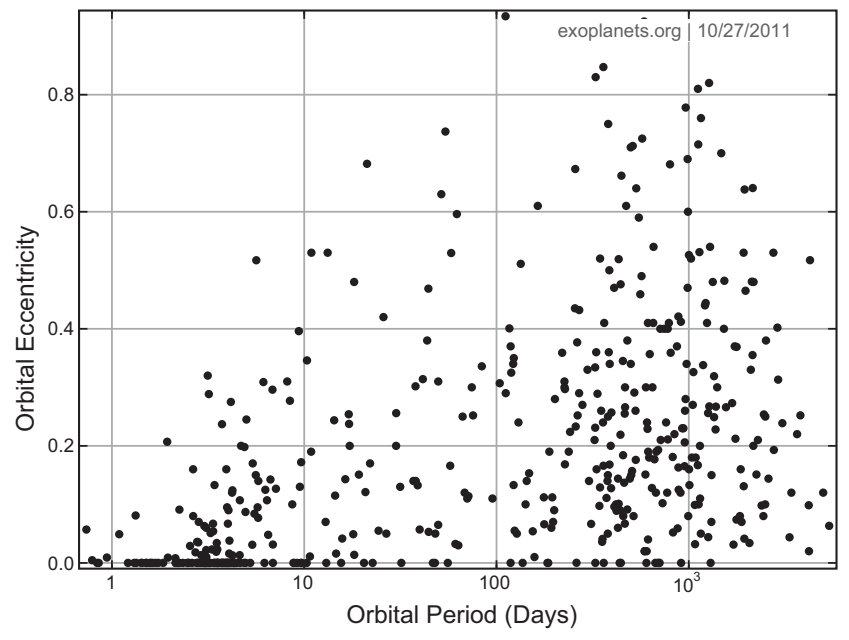

FIG. 2. Orbital eccentricity vs. period for known radial velocity planets.

\section{Extreme Planetary Orbits}

Here we describe cases where a planet moves in and out of the HZ due to the eccentricity of the orbit and the subsequent effects on habitability.

\subsection{Frequency of eccentric planets}

Among the surprises included in exoplanet discoveries is that of planets in highly eccentric orbits. Due to dynamical stability considerations, most of the very eccentric planets occupy single-planet systems. To show the frequency of these kinds of planetary orbits, we extracted data from the Exoplanet Orbit Database at exoplanets.org (Wright et al., 2010). This resource includes only those planets that have complete orbital solutions, which, at the time of writing, consists of 489 planets. Figure 2 plots the orbital eccentricity of these planets against the period. Of these, 187 (38\%) have eccentricities larger than 0.2 . There is a clear correlation with an increasing eccentricity dispersion with increasing period since short-period orbits have much smaller tidal circularization timescales (Goldreich and Soter, 1966). Figure 3 shows the distributions of planetary masses over this same period range. One may thus be tempted to conclude from this figure that eccentric planets tend to be of jovian mass and at large semimajor axis. There is a selection effect, however, such that smaller-mass planets at longer periods are more difficult to detect using radial velocity methods. Even so, these two figures do demonstrate that there are a large number of eccentric gas giants which fall within the expected period range for the $\mathrm{HZ}$ of main sequence stars.

\subsection{Orbital passage through the habitable zone}

There have been several interesting studies regarding the habitability of terrestrial-mass planets in eccentric orbits, such as that by Dressing et al. (2010). Kita et al. (2010) show the possible detrimental effects on terrestrial planet habitability when the planet is excited to an eccentric orbit by another companion in the system. Spiegel et al. (2010) consider the effect of transiently eccentric orbits on planet habitability. In particular, the work of Williams and Pollard 


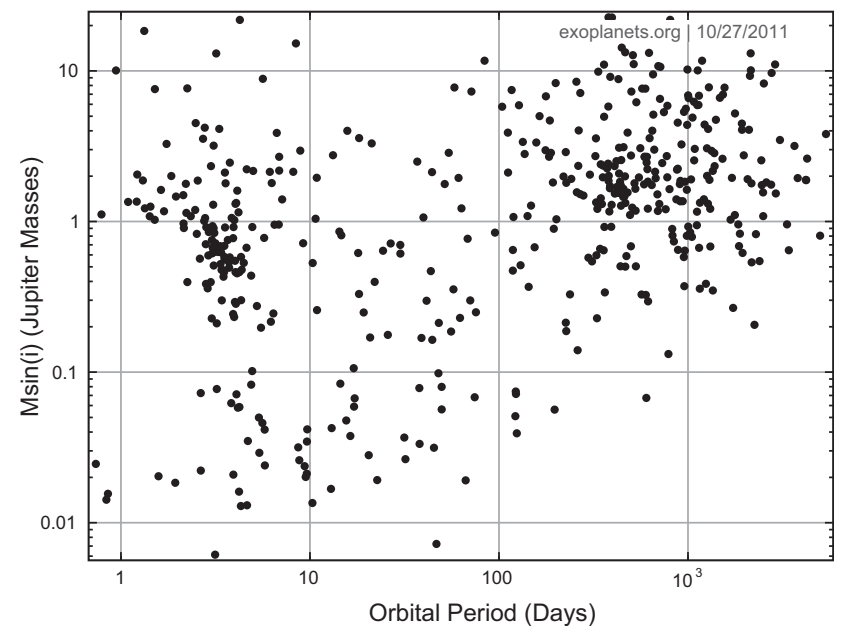

FIG. 3. Planetary mass vs. period for known radial velocity planets.

(2002) considers an orbit-averaged flux as a habitability diagnostic for eccentric orbits. We diverge from this metric since the average distance of the planet from the star is significantly farther than the difference between apastron and periastron; thus we use the percentage of time spent in the $\mathrm{HZ}$ where metabolic processes could proceed rather than relying on sustainable temperatures. However, the calculated equilibrium temperatures (as described in Section 2.1) make implicit assumptions regarding the response of the atmosphere to changes in flux. In reality, planetary temperatures at the surface and upper atmosphere are complicated functions of surface and atmospheric composition and dynamics as well as the long-term climate history and so can only be treated as a first-order approximation. See for example Williams and Kasting (1997), Spiegel et al. (2008, 2009), Kane and Gelino (2011), and references therein. Here we

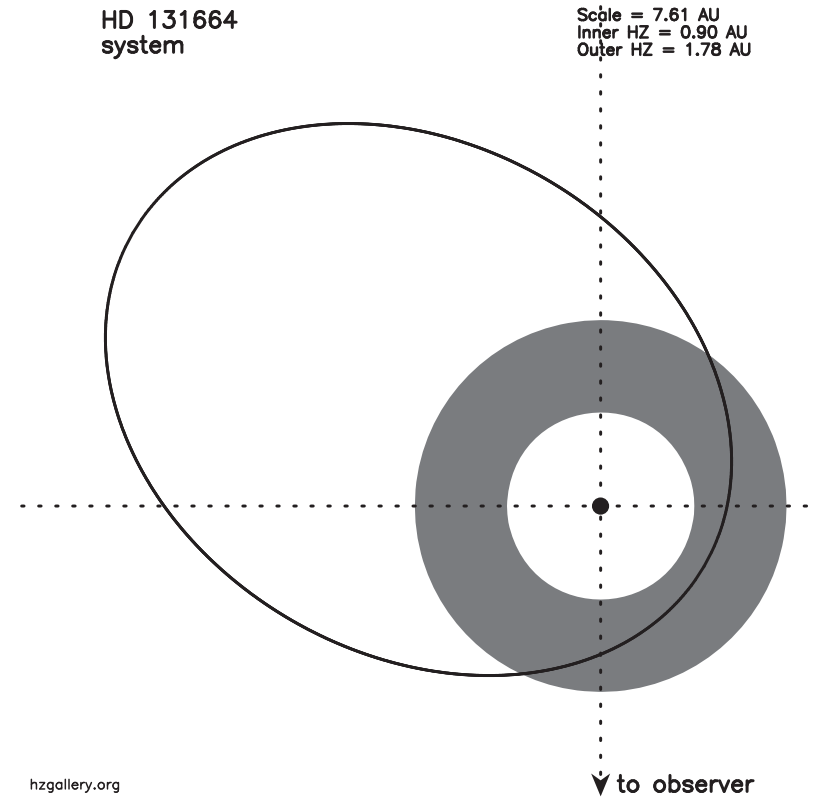

FIG. 4. The planetary orbit and HZ for the HD 131664 system.

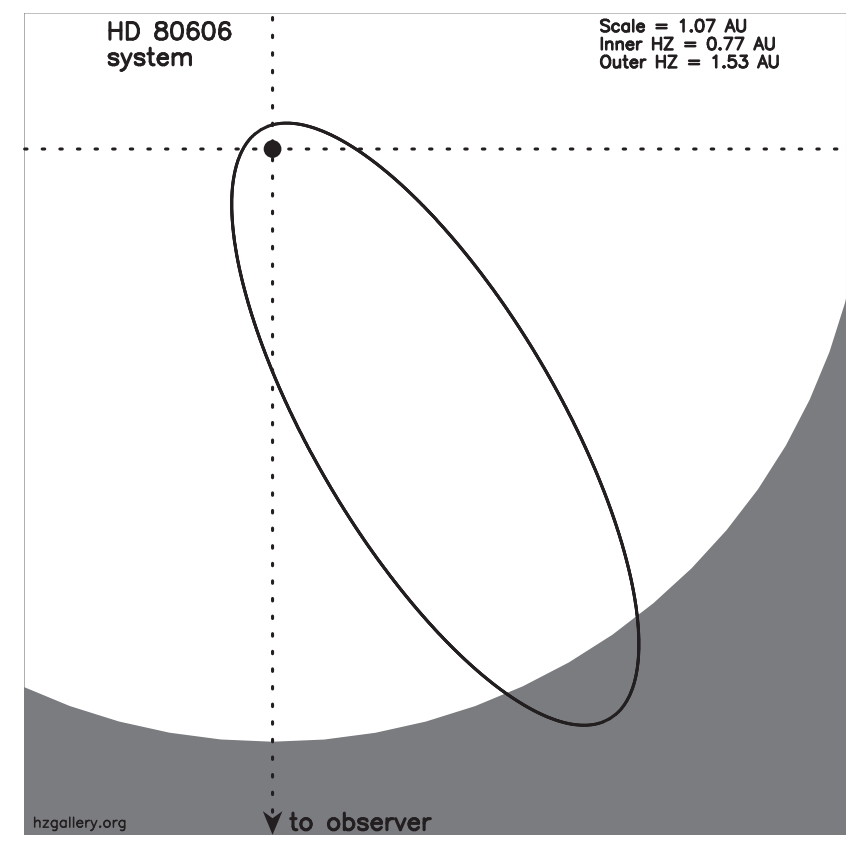

FIG. 5. The planetary orbit and HZ for the HD 80606 system.

briefly discuss two examples, and we refer the reader to Kane and Gelino $(2011,2012)$ and the Habitable Zone Gallery for more detailed information.

One important consideration is the orbital stability of exomoons as the parent planet passes through periastron. Hamilton and Burns (1992) showed that the Hill radius at periastron is a good representation of the stability zone for satellites of planets in highly eccentric orbits. The giant planets discussed here will have Hill radii that extend beyond Galilean moon analogues and thus could retain similar moons under these conditions.

In Figs. 4 and 5 we present two examples, one with periastron in the $\mathrm{HZ}$ and the other with apastron in the $\mathrm{HZ}$ (shown in gray). The planet orbiting HD 131664 is in a 1951day orbit and has an eccentricity of 0.64 . The minimum mass of the planet is 18 Jupiter masses, resulting in a Hill Sphere radius of $0.2 \mathrm{AU}$ (416 Jupiter radii) at periastron. The planet only spends $11 \%$ of the orbit in the HZ since it moves slowly near apastron. The calculated equilibrium temperature is 271 $\mathrm{K}$ at periastron and $127 \mathrm{~K}$ at apastron assuming a Bond albedo of 0 meaning that the planet and moons absorb $100 \%$ of the incident flux. Thus the planet and possible moons undergo long periods of hibernation conditions broken regularly with warmer habitable conditions (see Section 3.3). In fact the situation is improved for eccentric orbits through the extension of the outer HZ boundary as found by Williams and Pollard (2002) and Dressing et al. (2010). In contrast, the planet orbiting HD 80606 is in a 111-day orbit with an eccentricity of 0.93 and is known to both transit and be eclipsed by its host star (Laughlin et al., 2009). This planet has a mass of 3.9 Jupiter masses leading to a Hill Sphere radius of 0.0032 AU (6.7 Jupiter radii). In this case it is the apastron which lies within the HZ, where it spends $40 \%$ of the orbital phase. The temperature at apastron is predicted as $286 \mathrm{~K}$; however, the close encounter with the star at periastron causes temperatures to reach a scorching $1546 \mathrm{~K}$. This results in flash- 
heating of the upper atmosphere, which doubles in temperature in only 6 hours as it passes through periastron. Thus organisms could only survive such environments if deeply buried under sufficient protective layers. For both of these examples, regular intervals of habitability for the moons depend strongly on the surface conditions and their response to the change in temperatures. For example, a water-rich moon will require sufficient time to melt out of a snowball state to allow metabolic processes to continue.

\subsection{Hibernation and sterilization}

We now consider the survivability of extremophiles in these environments. A transition from liquid to frozen water and back again does not present an obstacle for most Earthbased microorganisms. It has been shown by de La Torre et al. (2010) that organisms such as lichens and bacteria can survive for periods of at least 10 days when exposed to the harsh conditions of outer space. Foucher et al. (2010) presented the results of atmosphere reentry experiments that demonstrate that microfossils can survive entry into Earth's atmosphere within sedimentary rock but that microorganisms require protection by at least $5 \mathrm{~cm}$ of rock for adequate shielding. During the time an exoplanet or exomoon spends exterior to the HZ, similar organisms can undergo metabolic slowdown for a period of hibernation without any immediate biological harm. The daily and annual biological clock we see exhibited by plants and cells on Earth could be adapted to different orbital durations to optimize time spent in favorable (habitable) conditions. Since we know that several terrestrial organisms can withstand substantial periods of time under extreme temperature conditions in combination with vacuum, UVC irradiation, and cosmic rays, then it is conceivable that such organisms could survive periastron flash-heating at a sufficient protective depth beneath the surface. In particular, oceans have a relatively high thermal inertia and could be maintained in liquid form even if the surface waters freeze over during the apastron winter. This can provide an environment where the annual range of temperatures is significantly less extreme than those experienced at the surface.

\section{Conclusions}

Although the concept of the HZ has been discussed for some time, it is only recently that sophisticated climate models are allowing concise quantification of this region. The study of exoplanetary atmospheres enables us to apply these concepts to known exosystems, even those with exoplanets in orbits which result in extreme temperature variations. A targeted search for exomoons in these environments may yield more surprises regarding what we consider "habitable."

\section{Acknowledgments}

The authors would like to thank Lisa Kaltenegger, Ravikumar Kopparapu, and René Demets for several useful discussions. We would also like to thank both the referees (Shawn Domagal-Goldman and anonymous) for their feedback, which greatly improved the manuscript. This research has made use of the Exoplanet Orbit Database and the Exoplanet Data Explorer at exoplanets.org. It has also made use of the Habitable Zone Gallery at hzgallery.org.

\section{Author Disclosure Statement}

No competing financial interests exist.

\section{Abbreviation}

$\mathrm{HZ}$, habitable zone.

\section{References}

Agol, E., Cowan, N.B., Knutson, H.A., Deming, D., Steffan, J.H., Henry, G.W., and Charbonneau, D. (2010) The climate of HD 189733b from fourteen transits and eclipses measured by Spitzer. Astrophys J 721:1861-1877.

Barnes, R., Jackson, B., Greenberg, R., and Raymond, S.N. (2009) Tidal limits to planetary habitability. Astrophys J 700:L30-L33.

Bean, J.L., Miller-Ricci Kempton, E., and Homeier, D. (2011) A ground-based transmission spectrum of the super-Earth exoplanet GJ 1214b. Nature 468:669-672.

Charbonneau, D., Allen, L.E., Megeath, S.T., Torres, G., Alonso, R., Brown, T.M., Gilliland, R.L., Latham, D.W., Mandushev, G., O'Donovan, F.T., and Sozzetti, A. (2005) Detection of thermal emission from an extrasolar planet. Astrophys J 626: 523-529.

Croll, B., Albert, L., Jayawardhana, R., Miller-Ricci Kempton, E., Fortney, J.J., Murray, N., and Neilson, H. (2011) Broadband transmission spectroscopy of the super-Earth GJ 1214b suggests a low mean molecular weight atmosphere. Astrophys J 736:78-88

de La Torre, R., Sancho, L.G., Horneck, G., de los Ríos, A., Wierzchos, J., Olsson-Francis, K., Cockell, C.S., Rettberg, P., Berger, T., de Vera, J.-P.P., Ott, S., Martinez Frías, J., Gonzalez Melendi, P., Mercedes Lucas, M., Reina, M., Pintado, A., and Demets, R. (2010) Survival of lichens and bacteria exposed to outer space conditions-results of the Lithopanspermia experiments. Icarus 208:735-748.

Dole, S.H. and Asimov, I. (1964) Planets for Man, Random House, New York.

Dressing, C.D., Spiegel, D.S., Scharf, C.A., Menou, K., and Raymond, S.N. (2010) Habitable climates: the influence of eccentricity. Astrophys J 721:1295-1307.

Foucher, F., Westall, F., Brandstätter, F., Demets, R., Parnell, J., Cockell, C.S., Edwards, H.G.M., Bény, J.-M., and Brack, A. (2010) Testing the survival of microfossils in artificial martian sedimentary meteorites during entry into Earth's atmosphere: the STONE 6 experiment. Icarus 207:616-630.

Goldreich, P. and Soter, S. (1966) Q in the Solar System. Icarus 5:375-389.

Hamilton, D.P. and Burns, J.A. (1992) Orbital stability zones about asteroids. II-The destabilizing effects of eccentric orbits and of solar radiation. Icarus 96:43-64.

Hart, M.H. (1979) Habitable zones about main sequences stars. Icarus 37:351-357.

Huang, S.-S. (1959) The problem of life in the Universe and the mode of star formation. Publ Astron Soc Pac 71:421-424.

Huang, S.-S. (1960) The sizes of habitable planets. Publ Astron Soc Pac 72:489-493.

Jones, B.W. and Sleep, P.N. (2010) Habitability of exoplanetary systems with planets observed in transit. Mon Not $R$ Astron Soc 407:1259-1267.

Kaltenegger, L. (2010) Characterizing habitable exomoons. Astrophys J 712:L125-L130.

Kaltenegger, L. and Sasselov, D. (2011) Exploring the habitable zone for Kepler planetary candidates. Astrophys J 736:L25-L30.

Kaltenegger, L., Udry, S., and Pepe, F. (2011) A habitable planet around HD 85512? arXiv:1108.3561v1. 
Kane, S.R. and Gelino, D.M. (2010) Photometric phase variations of long-period eccentric planets. Astrophys J 724:818-826.

Kane, S.R. and Gelino, D.M. (2011) Detectability of exoplanet periastron passage in the infrared. Astrophys J 741:52-60.

Kane, S.R. and Gelino, D.M. (2012) The habitable zone gallery. Publ Astron Soc Pac 124:323-328.

Kasting, J.F. (1988) Runaway and moist greenhouse atmospheres and the evolution of earth and Venus. Icarus 74:472-494.

Kasting, J.F. and Catling, D. (2003) Evolution of a habitable planet. Annu Rev Astron Astrophys 41:429-463.

Kasting, J.F., Whitmire, D.P., and Reynolds, R.T. (1993) Habitable zones around main sequence stars. Icarus 101:108-128.

Kipping, D.M., Fossey, S.J., and Campanella, G. (2009) On the detectability of habitable exomoons with Kepler-class photometry. Mon Not R Astron Soc 400:398-405.

Kipping, D.M., Bakos, G.A., Buchhave, L.A., Nesvorny, D., and Schmitt, A. (2012) The hunt for exomoons with Kepler (HEK): I. Description of a new observational project. Astrophys J 750: 115-133.

Kita, R., Rasio, F., and Takeda, G. (2010) Pervasive orbital eccentricities dictate the habitability of extrasolar Earths. Astrobiology 10:733-741.

Knutson, H.A., Charbonneau, D., Allen, L.E., Fortney, J.J., Agol, E., Cowan, N.B., Showman, A.P., Cooper, C.S., and Megeath, S.T. (2007) A map of the day-night contrast of the extrasolar planet HD 189733b. Nature 447:183-186.

Lopez, B., Schneider, J., and Danchi, W.C. (2005) Can life develop in the expanded habitable zones around red giant stars? Astrophys J 627:974-985.

Latham, D.W., Stefanik, R.P., Mazeh, T., Mayor, M., and Burki, G. (1989) The unseen companion of HD114762-a probable brown dwarf. Nature 339:38-40.

Laughlin, G., Deming, D., Langton, J., Kasen, D., Vogt, S., Butler, P., Rivera, E., and Meschiari, S. (2009) Rapid heating of the atmosphere of an extrasolar planet. Nature 457:562-564.

Mayor, M. and Queloz, D. (1995) A Jupiter-mass companion to a solar-type star. Nature 378:355-359.

Pollack, J.B. (1971) A nongrey calculation of the runaway greenhouse: implications for Venus' past and present. Icarus 14:295-306.

Porter, S.B. and Grundy, W.M. (2011) Post-capture evolution of potentially habitable exomoons. Astrophys J 736:L14-L19.

Richardson, L.J., Deming, D., Horning, K., Seager, S., and Harrington, J. (2007) A spectrum of an extrasolar planet. Nature 445:892-895.

Scharf, C.A. (2006) The potential for tidally heated icy and temperate moons around exoplanets. Astrophys J 648:11961205.
Selsis, F., Kasting, J.F., Levrard, B., Paillet, J., Ribas, I., and Delfosse, X. (2007) Habitable planets around the star Gliese 581? Astron Astrophys 476:1373-1387.

Spiegel, D.S., Menou, K., and Scharf, C.A. (2008) Habitable climates. Astrophys J 681:1609-1623.

Spiegel, D.S., Menou, K., and Scharf, C.A. (2009) Habitable climates: the influence of obliquity. Astrophys J 691:596-610.

Spiegel, D.S., Raymond, S.N., Dressing, C.D., Scharf, C.A., and Mitchell, J.L. (2010) Generalized Milankovitch cycles and longterm climatic habitability. Astrophys J 721:1308-1318.

Underwood, D.R., Jones, B.W., and Sleep, P.N. (2003) The evolution of habitable zones during stellar lifetimes and its implications on the search for extraterrestrial life. International Journal of Astrobiology 2:289-299.

von Paris, P., Gebauer, S., Godolt, M., Grenfell, J.L., Hedelt, P., Kitzmann, D., Patzer, A.B.C., Rauer, H., and Stracke, B. (2010) The extrasolar planet Gliese 581d: a potentially habitable planet? Astron Astrophys 522:23-33.

Williams, D.M. and Kasting, J.F. (1997) Habitable planets with high obliquities. Icarus 129:254-267.

Williams, D.M. and Pollard, D. (2002) Earth-like worlds on eccentric orbits: excursions beyond the habitable zone. International Journal of Astrobiology 1:61-69.

Williams, D.M., Kasting, J.F., and Wade, R.A. (1997) Habitable moons around extrasolar giant planets. Nature 385:234-236.

Wolszczan, A. and Frail, D.A. (1992) A planetary system around the millisecond pulsar PSR1257+12. Nature 355:145-147.

Wordsworth, R.D., Forget, F., Selsis, F., Millour, E., Charnay, B., and Madeleine, J.-P. (2011) Gliese 581d is the first discovered terrestrial-mass exoplanet in the habitable zone. Astrophys J 733:L48-L52.

Wright, J.T., Fakhouri, O., Marcy, G.W., Han, E., Feng, Y., Johnson, J.A., Howard, A.W., Fischer, D.A., Valenti, J.A., Anderson, J., and Piskunov, N. (2010) The Exoplanet Orbit Database. Publ Astron Soc Pac 123:412-422.

Address correspondence to: Stephen Kane NASA Exoplanet Science Institute Caltech MS 100-22

770 South Wilson Ave. Pasadena, CA 91125

E-mail: skane@ipac.caltech.edu

Submitted 1 December 2011 Accepted 10 May 2012 